\title{
Vaginal birth after Cesarean experience in Romania: A retrospective case-series study and online survey
}

\author{
ALEXANDRU CARAULEANU, INGRID ANDRADA TANASA, \\ DRAGOS NEMESCU, RALUCA HABA and DEMETRA SOCOLOV
}

Department of Obstetrics and Gynecology, 'Grigore T. Popa' University of Medicine and Pharmacy, 700115 Iasi, Romania

Received May 10, 2021; Accepted June 9, 2021

DOI: $10.3892 /$ etm.2021.10326

\begin{abstract}
The high Romanian Cesarean delivery rates have led to an increase in a women's desire to achieve vaginal birth after Cesarean delivery (VBAC), but certain impediments make this process difficult. We retrospectively evaluated the VBAC cases in a tertiary maternity hospital from Romania, and assessed the Romanian women's opinion about VBAC using a questionnaire that was distributed to three specific Facebook groups. We evaluated 63 patients who achieved VBAC between January 2014 and August 2020. In addition, an online survey with 41 open and closed questions assessed the participants' opinion regarding VBAC in Romania. The results are summarized and discussed. The results revealed that VBAC rates were under $1 \%$. The main observed complications that followed VBAC were postpartum hemorrhage $(15.8 \%)$, and uterine atony $(7.93 \%)$, while the most frequent types of lesions encountered after VBAC were cervical tear (12.6\%), followed by labial fissure $(9.5 \%)$ and vaginal tear $(7.9 \%)$. The patient's confidence in the health care system is low, and many women decide to try TOLAC at home. In conclusion, VBAC is an important topic in the context of high Romanian Cesarean rates, and the decision factors must provide consistent strategies that promote this mode of delivery.
\end{abstract}

\section{Introduction}

Cesarean delivery has become a prominent element in female obstetrical history, and two options emerge as solutions for

Correspondence to: Dr Ingrid Andrada Tanasa, Department of Obstetrics and Gynecology, 'Grigore T. Popa' University of Medicine and Pharmacy, 16 Universitatii Street, 700115 Iasi, Romania E-mail: tanasaingrid@yahoo.com

Abbreviations: ACOG, American College of Obstetricians and Gynecologists; CS, Cesarean section; ERCD, elective repeat Cesarean delivery; TOLAC, trial of labor after Cesarean; VBAC, vaginal birth after Cesarean

Key words: vaginal birth after Cesarean, trial of labor after Cesarean, birth care, uterine rupture, Cesarean section future births in these patients: Elective repeat Cesarean delivery (ERCD) or vaginal birth after Cesarean delivery (VBAC).

A woman's desire to achieve a VBAC frequently encounters obstacles that make this experience difficult. Whether we talk about the obstetrician's preferences for ERCD, lack of trained health care professionals, equipment, protocols and hospital facilities, or about legal and moral liabilities, the background for this problem is a complex one, and an in-depth analysis of all of these factors may provide evidence for further changes in paradigms.

VBAC rates across Europe vary from 20 to $55 \%(1,2)$, while in the United States of America (USA), the national VBAC rate was $13.3 \%$ in 2018 (3). A statistic concerning the VBAC rates in Romania for the purpose of this article could not be found.

In the VBAC equation, three main categories of actors are involved (the pregnant patient, the healthcare providers, and the healthcare managers), and each of them can influence the decisions regarding labor and the birth process.

Patient desire for achieving VBAC has been studied in a variety of articles. In a recent study by Attanasio et al (4), at 12 months postpartum, $45 \%$ percent of women who delivered by Cesarean their first birth preferred to have their next baby vaginally, and the percentage was higher among young women between 18 and 24 years of age (57.1\%), black women (75\%), and Latina women (54\%). In another study $39.5 \%$ of women preferred a VBAC in a subsequent pregnancy (5).

A cross-sectional web-survey identified as significant predictors for women choosing a VBAC as the following: i) Duration since previous birth in years $(\mathrm{P}=0.010)$; ii) having had midwifery care during pregnancy $(\mathrm{P}=0.029)$; iii) being advised by their healthcare provider to attempt a VBAC $(\mathrm{P}=0.001)$; iv) preference for VBAC during the third trimester of their pregnancy $(\mathrm{P}=0.001)$ and $\mathrm{v})$ wishing to let the child choose the moment of birth $(\mathrm{P}<0.001)(6)$.

Healthcare providers (obstetricians and midwives) are essential for a successful VBAC outcome, but only a few studies on the clinicians' views of VBAC have been conducted. In a qualitative study by Lundgren et al (7), the authors stated that the interviewed clinicians were 'highly positive about recommending VBAC', considered that 'an effective collaboration requires good communication between midwives and obstetricians', as well as 'working in accordance 
with a model and making agreements with the woman', and that 'the final decision should be made by a professional with medical knowledge'.

Another qualitative study indicated that the fear of liability, the convenience of having a Cesarean section (CS), and the marginalization of midwives led to VBAC avoidance by healthcare professionals (8).

The types of hospital (private or public, teaching or nonteaching) influence CS and VBAC rates, so that the private sector has higher Cesarean rates, and lower VBAC rates in contrast to public hospitals (9-11).

This article focuses on the first two mentioned categories of actors, the patient and the obstetricians, regarding the patient's perspective, as well as the obstetricians' experience with VBAC cases in Romania.

Specifically, the aims of this study were to retrospectively evaluate the six years of VBAC experience in a tertiary maternity hospital in Romania, and to assess the Romanian women's opinion about VBAC, using an online questionnaire distributed to specific Facebook groups.

\section{Subjects and methods}

The first objective of the present study was to retrospectively assess the VBAC cases that were admitted to a tertiary maternity hospital, 'Cuza Voda', Iasi, Romania, between January 2014 and August 2020. The study was reviewed and approved by the Institutional Ethics Committee of ' $\mathrm{Cuza}$ Voda' Maternity Hospital (approval no. 9836/09.09.2020). We included patients who had undergone one low-transverse previous CS that have successfully achieved VBAC, and we identified 67 cases, out of which two cases were excluded because the patients delivered in the ambulance or at home, and other two cases were excluded because the exact number of previous CS was unknown.

A total of 63 cases were analyzed, and relevant information on maternal and fetal parameters including outcome of the present pregnancy (age, interval between admission to the hospital and delivery, present pregnancy and previous CS, place, indication, of previous CS, mode of delivery in the present pregnancy, maternal disorders, and maternal and perinatal outcome) were collected in structured pro-forma, entered in Microsoft Office Excel format. Statistical analysis was performed using SPSS software (version 24.0) (IBM Corp.). All values are expressed in the form of proportion and percentages.

Informed consent was obtained from all participants included in the study. All methods were carried out in accordance with relevant guidelines and regulations. The study was reviewed and approved by the Institutional Ethics Committee from 'Cuza Voda' Maternity Hospital, composed of Professor Doctor Mihaela Grigore, and Assistant Professor Doctor Catalin Mihaila.

The second objective of this study was to assess the women's perception of the VBAC experience in Romania. Data were collected using a google form developed by the investigator and entitled: 'A survey for evaluating the Romanian patient's opinions regarding vaginal delivery after Cesarean section (VBAC)' (Data S1), that was published on three Facebook groups between August 29 and September 5, 2020. The questionnaire consisted of 41 questions that addressed maternal characteristics (age, living environment, level of education), number of vaginal or Cesarean deliveries, indication for the first Cesarean delivery, gestational age at birth, VBAC topic (closed and open questions for identifying the women's opinions regarding the relationship with their obstetrician, relatives, number of successful VBACs and complications associated with them), type of hospital chosen for birth, and a series of open questions that allowed respondents to express their feelings regarding the vaginal, Cesarean delivery or VBAC, and to suggest changes for improvement of the Romanian maternity care system. Group administrators approved the posting of the questionnaire, and participants were informed that their anonymous responses would be published in this study. To protect the participant's identity, no names or medical identification numbers were recorded.

Data collected were translated from the Romanian language into standard English, and structured into a Microsoft Office Excel database. Statistical analysis was performed using SPSS software (version 24.0) (IBM Corp.). All values are expressed in the form of percentages.

\section{Results}

VBAC experience in one tertiary maternity center. A total of 63 cases of VBAC were included in our study. The mean age of the patients was 30.52 years [minimum (min.) age, 19 years; maximum (max.) age, 42 years; standard deviation (SD), 5.645]. In our selected cases, 40 patients (63\%) received prenatal care, $21(33 \%)$ patients did not receive any prenatal care, and 2 patients (4\%) were partially examined by family doctors or obstetricians during their pregnancy. The most frequent maternal comorbidities were vaginal infection with Escherichia coli (5 cases, 7\%) or Streptococcus agalactiae ( 5 cases, $7 \%$ ), feto-maternal rhesus incompatibility (5 cases, $7 \%$ ), followed by cervical incompetence ( 3 cases, $4 \%$ ); 31 pregnant patients $(42 \%)$ had no comorbidities.

The mean admission to delivery time interval expressed in days was 0.97, with the longest hospitalization of 22 days. All 63 cases had one low previous transverse CS and achieved VBAC. A total of 27 patients $(42.8 \%)$ had ruptured membranes before VBAC.

The number of successful cases of VBAC was 4 in 2014, 10 in 2015, 4 in 2016, 7 in 2017, 12 in 2018, 17 in 2019, and 9 in 2020 (until 05/09/2020). The main doctors that assisted VBAC were AC (28 births), MT (5 births) and MH (4 births), while other doctors assisted one or two VBACs.

Prophylactic episiotomy was performed in 38 cases (60.3\%), and instrumental delivery was indicated in 8 cases (12.6\%): 7 vacuum extractions, and one forceps application.

The mean gestational age at birth was 37.25 weeks of gestation. The smallest gestational age was 27 weeks, while the maximum gestational age at birth in our cases was 42 weeks. A total of 15 preterm births were identified: 3 cases (20\%) of extreme preterm births, 5 cases $(33 \%)$ of very preterm births, and 7 cases $(47 \%)$ of late-term births. Only one case $(1.5 \%)$ of post-term birth at 42 gestational weeks, and 4 other cases $(6.3 \%)$ of late-term births at 41 weeks were recorded.

The male:female ratio for newborns was 1.73 , with a mean birth weight of 2,956.03 g, respectively a mean Apgar score at $1 \mathrm{~min}$ of 7.63 . There were 4 antepartum fetal deaths. 
The main complications that followed VBAC were postpartum hemorrhage (10 cases, 15.8\%) and uterine atony (5 cases, $7.93 \%$ ), which required manual removal of the placenta, instrumental and manual control of the uterine cavity. Postpartum transfusion of two units of blood were required in two cases of VBAC with vacuum extraction that had cervical tears.

The most frequent type of lesion encountered after VBAC was cervical tear ( 8 cases, $12.6 \%$ ), followed by labial fissure (6 cases, $9.5 \%$ ), vaginal tear (5 cases, $7.9 \%$ ), first- and second-degree perineal tear ( 3 cases each, $4.7 \%$ ). A case with type II diabetes became unbalanced after VBAC and required transfer to a multidisciplinary hospital.

Antibiotic therapy was recommended for 33 patients (52.3\%). Intravenous antibiotic therapy consisted of ampicillin $1 \mathrm{~g}$ q6h (15 patients), cefotaxime $2 \mathrm{~g}$ bid (4 patients), amoxicillin-clavulanic acid $1 \mathrm{~g}$ bid (5 patients), ampicillin $1.5 \mathrm{~g}$ bid (2 patients), metronidazole $500 \mathrm{mg}$ bid (1 patient, in combination with cefotaxime) and gentamicin $1.5 \mathrm{~g} \mathrm{q} 8 \mathrm{~h}$ (1 patient).

Venofer (iron sucrose) $200 \mathrm{mg}$ was administered for 6 patients $(9.5 \%)$ who developed moderate anemia (hemoglobin $<10 \mathrm{~g} / \mathrm{dl})$.

AntiD immunoglobulin (Rhophylac, $300 \mu \mathrm{g}$ ) was administered in all cases with feto-maternal Rh incompatibility, and ablactation with either cabergoline $(1 \mathrm{mg})$ or bromocriptine $(2.5 \mathrm{mg})$ was offered for those patients with antepartum fetal death.

The Romanian women's perspective concerning VBAC. The second objective of our study was to assess, through an online survey, the Romanian women's opinions regarding VBAC. A total of 394 women responded to this online survey and 351 answers were validated, translated from Romanian language to standard English, and implemented into our database.

We separated the responders into four groups: Group 1, women who delivered vaginally; group 2 , women who delivered through CS; group 3, women who had both vaginal and Cesarean delivery in their obstetric history; and group 4, women who experienced VBAC. Group 1 consisted of 183 respondents, with a mean age of 28.21 years (max. 37 years; min. 18 years; SD 5.787). As for the living environment, 92 women $(50.3 \%)$ lived in urban areas, while 91 women lived $(49.7 \%)$ in rural areas. The educational background was mainly academic tertiary education (114 cases, $62.3 \%$ ), followed by post high school education (44 cases, 24\%), high school (20 cases, $10.9 \%)$, primary school ( 3 cases, $1.6 \%$ ), and secondary school (2 cases, $1.1 \%)$. The mean of vaginal births was 1.87 (min. 1; max. 5; SD 0.801), and the mean gestational age for birth was 38.85 ( $\min .37$; max. 41; SD 0.745). The vast majority of the respondents (174 cases, $95.1 \%$ ) declared that the mode of delivery was established after consulting with the obstetrician. Moreover, 120 women $(65.6 \%)$ declared that they received enough support from their obstetrician regarding vaginal delivery, in contrast to 41 women (22.4\%) who declared no support from their obstetrician, and 22 cases (12\%) that were partially supported in their decision for vaginal delivery. A total of $45.4 \%$ ( 83 cases) of the respondents suffered from a form of obstetrical violence, and most of them delivered in public health facilities: Local hospitals 130 cases
(71\%), university hospitals 50 cases (27.3\%), while another two women (1.1\%) delivered at private hospitals, and one woman $(0.5 \%)$ delivered at home.

The mean age of the second group which comprised 111 women was 27.5 years (min. 18; max. 40; SD 5.298), most of whom lived in the urban area (78 cases, $70 \%$ ), and had completed academic studies (77 women, 69.3\%). These women had a mean number of Cesarean deliveries of 1.33. The main indications for the first CS were placenta praevia (28 cases, 25.2\%), and obstructed labor (14 cases, 12.6\%). The mean gestational age for the first CS was 38.4 weeks (min. 25; max. 42; SD 2.2). A total of $85.6 \%$ of the women (95 cases) stated that the mode of delivery was established after consulting with their obstetrician, while $44.1 \%$ ( 49 women) declared that they had discussed a birth plan with their obstetrician, and only in 34 cases was that plan fully respected. In addition, $36.9 \%$ (41 cases) suffered from a form of obstetrical violence during labor and delivery. The local hospital was the most frequent healthcare facility for Cesarean delivery (54 cases, $48.6 \%$ ), while the private sector attracted 9 women $(8.1 \%)$.

Most women (101 cases, $91 \%$ ) declared that would like to try the VBAC experience, but only $36.9 \%$ (41 cases) received full support from their obstetrician regarding a possible vaginal delivery. Moreover, $89.2 \%$ of participants (99 cases) consider that their doctor would not support a VBAC attempt. At the same time, $96.3 \%$ of the women (107 cases) believed that the private health sector did not support VBAC. When asked if they were willing to go to another city for pursuing VBAC, $82 \%$ (91 cases) of them offered a negative response, while others preferred Bucharest (7 responses out of 17) for the VBAC attempt; $80.7 \%$ of patients (21 answers out of 26) would change their obstetrician if he/she would not support VBAC. The general opinion was that Romanian doctors were not open to VBAC (89 opinions, $80.2 \%$ ).

Only $18.9 \%$ of the women (21 cases) were supported by a doula during pregnancy or labor. The majority of hospitals did not allow access of the partners, doulas or other supporting persons in the maternity ward during labor and delivery.

The third group consisted of 46 patients with a mean age of 29.87 years (min. 20; max. 37; SD 4.796), most of whom lived in urban areas (30 cases, 65.2\%) and had a university degree (31 cases, 67.4\%). The mean number of vaginal births was 1.54 (min. 1; max. 12; SD 1.683), and the mean number of Cesarean deliveries was 1.22 (min. 1; max. 3; SD 0.467), while the mean gestational age for vaginal birth was 38.74 weeks (min. 29; max. 41; SD 1.612), and the mean gestational age for Cesarean deliveries was 38.41 weeks (min. 34; max. 41; SD 1.275). The main declared reasons for performing the CS were meconial liquor (6 cases, 13\%), and cephalopelvic disproportion ( 5 cases, $10.9 \%$ ), followed by breech presentation, obstructed labor, placenta praevia and unknown indication (4 cases each, 8.7\%). Most of them stated that the mode of delivery was established after consulting with them (37 women, 80.4\%). Only 16 women (34.8\%) discussed about a birth plan with their obstetrician, which was totally respected in 10 cases $(21.7 \%)$.

Local hospital was preferred for $67.4 \%$ of natural births (31 cases), and for $56.5 \%$ ( 26 cases of Cesarean deliveries). Only one natural delivery (2.2\%), and two Cesarean deliveries (4.3\%) occurred in a private hospital; $37 \%$ (17 cases) did suffer from a form of obstetrical violence during their delivery. 
All women in this group wanted to try a different approach of vaginal delivery, and the most preferred one was different labor positions (37 women, 80.4\%). Prayer (15 cases, 32.6\%), dance (6 cases, 13\%), essential oils (5 cases, 10.9\%), and breathing control (4 cases, $8.7 \%$ ) were the most frequently used techniques for relaxation and concentration during labor.

Ten women $(21.7 \%)$ in this group were supported by a doula during their pregnancy and labor, and in 7 cases the hospital allowed access for partner, doula or other persons in the labor ward $(15.2 \%)$.

When asked if they would like to achieve VBAC in further pregnancies, most of them offered an affirmative response (40 cases, $87 \%$ ), but $89.1 \%$ (41 cases) of women believed that their obstetrician would not support a TOLAC, and that Romanian obstetricians were not open to VBAC (34 women, $73.9 \%)$. The majority of respondents $(87 \%, 40$ cases) believed that the private health sector of Romania does not support VBAC.

Moreover, $82.6 \%$ (38 cases) would not travel to another city in order to achieve VBAC, while only 8 women declared in favor of choosing another city for this purpose, out of which 4 women preferred Bucharest (6 answers).

Thirteen women who achieved VBAC responded to our survey, and formed the fourth group of this study. The mean age in this group was 33.77 years (min. 25; max. 43; SD 4.711). Most respondents came from urban areas (11 women, 84.6\%), and had a university degree (12 women, 92.3\%).

A total of $84.6 \%$ (11 women) of them had one previous CS. The gestational age of the first CS was 39 and 40 weeks (5 cases each, 38.5\%), and none delivered preterm. The main indications for the first CS were transverse presentation (4 cases, 30.8\%), acute fetal distress, and obstructed labor ( 2 cases each, 15.4\%). The mode of delivery was established after consulting with the obstetrician in $69.2 \%$ of the cases (9 women).

Only one woman achieved 3 VBACs $(7.7 \%)$, the rest of them (12 cases, $92.3 \%$ ) had only one VBAC in their obstetrical history. Three women $(23.1 \%)$ had a previous vaginal birth before attempting VBAC. The majority of women achieved VBAC at home (7 cases, $53.8 \%$ ), while 5 women delivered at university hospitals (38.5\%), and only one delivered at a local hospital $(7.7 \%)$. No personal obstetrician was present during VBAC in 8 cases $(61.5 \%)$ because 7 women delivered at home, and in another case, due to the SARS-CoV-2 pandemic, the obstetrician was not allowed to come from home, and assist the VBAC case. All women had a successful VBAC, without complications; $53.8 \%$ of the women ( 7 cases) discussed a birth plan with their obstetrician, but in 5 cases it was not respected.

During their labor and delivery (vaginal, Cesarean, or VBAC), $84.6 \%$ (11 women) suffered from a form of obstetrical violence, and $76.9 \%$ (10 cases) felt stigmatization from others when they expressed their desire for VBAC.

Twelve women $(92.3 \%)$ wanted to attempt VBAC one more time, while one woman $(7.7 \%)$ was undecided. Most of the women (9 answers, 69.2\%) considered that their obstetrician would not support another VBAC attempt. Moreover, all of them believed that Romanian obstetricians do not support VBAC, while 9 women $(69.2 \%)$ claimed the same idea for the private health sector, and declared that they would change the obstetrician if he/she would not support the VBAC attempt.
When asked if they considered traveling to another city in order to achieve VBAC, 8 women (61.5\%) admitted this option, and they expressed only two options: Targu Mures (4 answers, $30.8 \%$ ), and Bucharest (3 answers, 23.1\%).

Most women in this group (12 cases, $92.3 \%$ ) would like to experiment with alternative ways of vaginal delivery, and were mainly interested in different labor positions (6 answers, 46.2\%), and water birth (5 answers, 38.5\%).

Prayer (4 answers, 30.8\%) and the control of breathing ( 3 answers, $23.1 \%$ ) were the main relaxation and concentration techniques used during labor for this group.

Support from a doula was received during pregnancy or labor in 4 cases $(30.8 \%)$, and in only one case $(7.7 \%)$ the hospital allowed access to the partner, doula or other persons in the labor room.

When asked to describe the Cesarean delivery experience, the vast majority of women used terms such as 'terrible', 'horrible', 'traumatizing', 'awful', 'terrifying', and 'difficult'.

As for the vaginal delivery, the terms used by women were 'wonderful', 'difficult', 'intense', and 'fulfilling'.

The VBAC experience was described as 'superb', 'fulfilling', 'healing', 'divine', 'wonderful', and 'stressful'.

The general suggestions recorded through this survey can be summarized as follows: a) To promote vaginal birth and VBAC; b) to ensure that obstetricians and midwives know and apply the VBAC protocols; c) to respect the patient's autonomy, and to allow different birthing positions, water birth, labor analgesia with nitrous oxide or epidural; d) to update the hospital protocols regarding birth, and to reduce the rate of $\mathrm{CS}$; e) to allow access of partners, doulas or other support persons into the labor ward; f) to respect the 'magic hour', and to delay cord clamping until it is pulseless; g) to implement the rooming in system on a national level; h) to promote patient education about pregnancy, labor, birth, and breastfeeding through prenatal courses and breastfeeding consultants; i) to change the attitude of obstetricians and midwives towards patients so that respect, patience, and support become the key points of the patient-healthcare professional relationship.

\section{Discussion}

The first objective of the present study was to retrospectively assess the VBAC cases that were admitted to a tertiary maternity hospital in Romania, between January 2014 and August 2020.

An increasing trend in the number of VBACs was observed, from 4 births in 2014 to 17 births in 2019, but in this tertiary center approximately 7,000 births take place annually, so the VBAC rate is less than $1 \%$. Since we could not find a statistic that outlines the VBAC rate in Romania, we concluded that this rate is extremely low compared to other European tertiary centers, where the VBAC rate is between 20 and $55 \%(1,2)$. Moreover, only one doctor, who is also the main author of this article, assisted the majority of VBAC cases in this center.

All 63 patients had a low transverse CS in their obstetrical history, and most of them received prenatal care. The indication for the Cesarean deliveries could not be established based on available medical records.

Prophylactic episiotomy was performed in the majority of cases, while for fetal extraction 7 vacuum and one forceps 
application were needed. Although, prophylactic episiotomy is considered by some women a form of obstetrical violence, and its utility was questioned in many studies (12-14), in our center it is a common practice, especially for women without previous vaginal births.

The mean gestational age at birth was 37.25 and the mean Apgar score at one minute was 7.63. There were 4 antepartum fetal deaths.

The main observed complications that followed VBAC were postpartum hemorrhage (15.8\%) and uterine atony (7.93\%). In a cross-sectional study (15), the incidence of post-partum hemorrhage was $2.7 \%$ of woman with successful VBAC, a result that was much lower than ours. The reasons for this observed difference may consist of instrumental delivery, lack of oxytocin infusion, or independent risk factors for postpartum hemorrhage.

In a study by Pont et al (16), the authors concluded that the risk of transfusion was higher for women attempting vaginal birth after a primary CS than after a planned repeat Cesarean. In our study, the necessary of blood transfusion was small, and was required in two cases of VBAC with vacuum extraction that had cervical tears.

In 2019, the American College of Obstetricians and Gynecologists (ACOG) emitted a practice bulletin that appreciated the risk of uterine rupture at $0.71 \%$ after failed TOLAC (17). In our study, no uterine rupture was detected.

The most frequent type of lesion encountered after VBAC was cervical tear $(12.6 \%)$, followed by labial fissure (9.5\%), and vaginal tear (5 cases, $7.9 \%$ ). In a retrospective cohort study (18), the authors determined that women in the VBAC groups (first VBAC, all VBAC) were more likely to have a third- or fourth-degree perineal tear $(5.6 \%, 7.42 \%)$, but in our study no such lesions were observed.

Intravenous prophylactic antibiotic therapy was recommended in $52.3 \%$ cases and consisted of penicillin, third generation cephalosporins, nitroimidazole or aminoglycoside.

Absence of a control group was the major limitation of our study, along with the small number of cases. But this is explained by the limited experience of Romanian obstetricians with VBAC cases, even though this center serves an important region of the country, where more than 4,000,000 people live. Longer studies with larger sample size might result in more accurate findings. One other probable limitation of our study was its design; our project was conducted in an academic hospital, in which supervision of experienced obstetricians can influence the incidence of maternal and neonatal complications.

The second objective of this study was to assess the women's perception of the VBAC experience in Romania using an online survey disseminated on three Facebook groups.

All respondents were adults, with a mean age of 28.21 (first group), 27.5 (second group), 29.7 (third group), and 33.77 (fourth group) years. The majority of women in these groups lived in urban areas, and had a university degree.

The vast majority of the respondents declared that the mode of delivery was established after consulting with the obstetrician.

Our survey showed alarming statistics concerning the obstetrical violence that was almost double for the VBAC group; $45.4 \%$ (first group), 36.9\% (second group), 37\% (third group), and $84.6 \%$ (fourth group) of the respondents suffered from a form of obstetrical violence. Although one may argue that these groups are unequal in number, the identified percentages raise an important topic for debate, and, more important, for a change in healthcare providers' attitude. The respect for the patients' autonomy must be primordial and unequivocal.

While for the first three groups, local hospital, followed by university hospital, were the main facilities used for birth, $53.8 \%$ VBAC group participants delivered at home, without an obstetrician. These findings may indicate that women who want to achieve VBAC tend to avoid giving birth at a Romanian healthcare facility, and prefer the comfort of their home. The reasons that may explain this situation are various, from pressure for ERCD exerted by healthcare providers to material shortcomings that one may find in the public hospitals.

The private health facilities were chosen by few respondents in all groups, and the vast majority of women consider that the private health sector does not support VBAC. This opinion is supported by studies that outlines the low VBAC rates in private hospitals $(19,20)$.

Another interesting finding is that placenta praevia was the main indication for the first CS in the second group (25.2\%), while in the third group it accounted for $8.7 \%$ of total indications for Cesarean delivery, and occupying the third position among indications.

In a study by Kollmann et al, the incidence of placenta praevia was only $0.15 \%$ (21), while Cresswell and colleagues estimated the prevalence of placenta praevia at 3.6 per 1,000 pregnancies for Europe and 2.9 per 1,000 pregnancies for North America (22). We hypothesize that the high percentage of this indication among groups is due to the fact that it constitutes a perfect back-up for obstetricians, who want to justify the Cesarean intervention, so that if no other sonographic finding is attached to the patient's medical record, the only evidence is removed during surgery, and no one can prove otherwise.

Only one woman achieved 3 VBACs, while the rest of the women (92.3\%) had only one VBAC in their obstetrical history. Previous vaginal birth before attempting VBAC is a powerful predictor of success $(23,24)$, and in the fourth group $23.1 \%$ of women had this feature.

No personal obstetrician was present during VBAC in 8 cases because 7 women delivered at home, and in another case, due to the SARS-CoV-2 pandemic, the obstetrician was not allowed to come from home, and assist the VBAC case. Until the SARS-CoV-2 pandemic, it was a common practice for obstetricians in Romania to come from home and assist their cases, but new regulations prohibited this situation, so that many women who wanted to achieve VBAC were assisted by the on-call doctor, who frequently did not support this type of delivery. This led to a further reduction in the VBAC rate in Romania.

All women had a successful VBAC, without declared complications which can be partly explained by the fact that most of them delivered at home. In a study by Rowe et al (25), the authors found higher vaginal birth rates in planned VBAC at home vs. in obstetrical units, but 2-3\% adverse outcomes, and high transfer rate. Another study by Macdorman et al (26), stated that planned home VBACs was associated with a lower risk profile than hospital VBACs.

Most women from all groups considered that their obstetrician would not support a VBAC attempt, and that 
Romanian obstetricians do not generally support VBAC. These opinions strongly suggest a low level of confidence in the healthcare professionals regarding their willingness to promote this mode of delivery.

Prayer and breathing control were the preferred methods for relaxation and concentration during labor and delivery, and this aspect can be easily explained by the fact that Romania is an orthodox country, so that religious practices are common in believers' lives.

When asked to describe the birth experience, women used positive terms for describing VBAC, and mainly negative terms for describing Cesarean delivery. This reflects two different perspectives over birth experience that need to be recognized, and in the case of Cesarean deliveries, improved.

The women's general suggestions regarding VBAC and birth experience in Romania addressed the main problems of this healthcare system: Lack of VBAC promotion, poorly trained healthcare professionals, and shortcomings of the birth practices in hospitals around the country.

In conclusion, the VBAC rates were low in the tertiary maternity hospital included in the study, and few obstetricians were involved in the processes of prenatal care, and birth assistance of such cases.

The main observed complications that followed VBAC were postpartum hemorrhage and uterine atony, while the most frequent types of lesions encountered after VBAC were cervical tear, followed by labial fissure and vaginal tear.

Romanian women with a previous Cesarean intervention in their obstetrical history are willing to achieve VBAC, but the confidence in the health care system is low, and many of them decide to give birth at home.

Improvements can be made, beginning with an attitude shift towards respect of patient autonomy, continuing with improvement of health care facilities and personnel training, and finishing with a change of paradigm for the entire Romanian health care system.

\section{Acknowledgements}

Not applicable.

\section{Funding}

No funding was received.

\section{Availability of data and materials}

The data used to support the findings of this study are available from the corresponding author upon reasonable request.

\section{Authors' contributions}

$\mathrm{AC}$ and IAT were responsible for conception and design. $\mathrm{DS}$, and RH were responsible for data acquisition. IAT was responsible for data analysis, statistical analysis, literature search, manuscript preparation, and manuscript editing. AC, DN, DS, and IAT were responsible for interpretation of the data. RH and IAT drafted the article; AC and DS revised it critically for important intellectual content. All authors gave the final approval of the version submitted for publication.
All authors read and approved the final manuscript and agreed to be accountable for all aspects of the work in ensuring that questions related to the accuracy or integrity of any part of the work are appropriately investigated and resolved.

\section{Ethics approval and consent to participate}

The study was reviewed and approved by the Institutional Ethics Committee from 'Cuza Voda' Maternity Hospital (Approval no. 9836/09.09.2020). The committee that approved the research was composed of Professor Doctor Mihaela Grigore and Assistant Professor Doctor Catalin Mihaila. Informed consent was obtained from all participants and/or their legal guardians included in the study. All research was performed in accordance with relevant guidelines/regulations.

\section{Patient consent for publication}

Not applicable.

\section{Competing interests}

The authors declare that they have no competing interests.

\section{References}

1. Lundgren I, Smith V, Nilsson C, Vehvilainen-Julkunen K, Nicoletti J, Devane D, Bernloehr A, van Limbeek E, Lalor J and Begley C: Clinician-centred interventions to increase vaginal birth after caesarean section (VBAC): A systematic review. BMC Pregnancy Childbirth 15: 16, 2015.

2. EURO-PERISTAT Project with SCPE and EUROCAT: European Perinatal Health Report. The health and care of pregnant women and babies in Europe in 2010. www.europeristat.com. Accessed 27 May, 2013 Accessed September 18, 2020.

3. Osterman MJK: Recent trends in vafginal birth after cesarean delivery: United States, 2016-2018. NCHS Data Brief, No. 359, March 2020. https://www.cdc.gov/nchs/data/databriefs/db359-h. pdf. Accessed September 18, 2020.

4. Attanasio LB, Kozhimannil KB and Kjerulff KH: Women's preference for vaginal birth after a first delivery by cesarean. Birth 46: 51-60, 2019.

5. Ryan G, O Doherty KC, Devane D, McAuliffe F and Morrison J: Questionnaire survey on women's views after a first caesarean delivery in two tertiary centres in Ireland and their preference for involvement in a future randomised trial on mode of birth. BMJ Open 9: 031766, 2019.

6. Bonzon M, Gross MM, Karch A and Grylka-Baeschlin S: Deciding on the mode of birth after a previous caesarean section-an online survey investigating women's preferences in Western Switzerland. Midwifery 50: 219-227, 2017.

7. Lundgren I, van Limbeek E, Vehvilainen-Julkunen $\mathrm{K}$ and Nilsson C: Clinicians' views of factors of importance for improving the rate of VBAC (vaginal birth after caesarean section): A qualitative study from countries with high VBAC rates. BMC Pregnancy Childbirth 15: 196, 2015.

8. Cox KJ: Providers' perspectives on the vaginal birth after cesarean guidelines in Florida, United States: A qualitative study. BMC Pregnancy Childbirth 11: 72, 2011.

9. Bailit JL, Dooley SL and Peaceman AN: Risk adjustment for interhospital comparison of primary cesarean rates. Obstet Gynecol 93: 1025-1030, 1999.

10. MacDorman MF, Menacker F and Declercq E: Cesarean birth in the United States: Epidemiology, trends, and outcomes. Clin Perinatol 35: 293-307, v, 2008.

11. DeFranco EA, Rampersad R, Atkins KL, Odibo AO, Stevens EJ, Peipert JF, Stamilio DM and Macones GA: Do vaginal birth after cesarean outcomes differ based on hospital setting? Am J Obstet Gynecol 197: 400.e1-e6, 2007. 
12. Tandon AN and Dalal ARA: A randomized, open-labelled, interventional study to evaluate the incidence of infection with or without use of prophylactic antibiotics in patients of episiotomy in a normal vaginal delivery. J Obstet Gynaecol India 68: 294-299, 2018.

13. Nalbanski A: Prophylactic episiotomy-a historical heritage with no medical foundation? Akush Ginekol (Sofia) 48: 36-38, 2009 (In Bulgarian).

14. Steiner N, Weintraub AY, Wiznitzer A, Sergienko R and Sheiner E: Episiotomy: The final cut? Arch Gynecol Obstet 286 $1369-1373,2012$

15. Mirteymouri M, Ayati S, Pourali L, Mahmoodinia M and Mahmoodinia M: Evaluation of maternal-neonatal outcomes in vaginal birth after cesarean delivery referred to maternity of academic hospitals. J Family Reprod Health 10: 206-210, 2016.

16. Pont S, Austin K, Ibiebele I, Torvaldsen S, Patterson J and Ford J: Blood transfusion following intended vaginal birth after cesarean vs. elective repeat cesarean section in women with a prior primary cesarean: A population-based record linkage study. Acta Obstet Gynecol Scand 98: 382-389, 2019.

17. ACOG practice bulletin No. 205: Vaginal birth after cesarean delivery. Obstet Gynecol 133: e110-e127, 2019.

18. Nettle JAS, Mcnamara HC and Du Plessis JM: Perineal trauma with vaginal birth after a previous caesarean section: A retrospective cohort study. Aust N Z J Obstet Gynaecol 59: 346-350, 2019.

19. Colais P, Bontempi K, Pinnarelli L, Piscicelli C, Mappa I, Fusco D and Davoli M: Vaginal birth after caesarean birth in Italy: Variations among areas of residence and hospitals. BMC Pregnancy Childbirth 18: 383, 2018.
20. Ying $Y H$, Linn $G$ and Chang $K$ : Vaginal birth after cesarean section in Taiwan: A population-based study. J Clin Med 8: 1203, 2019.

21. Kollmann M, Gaulhofer J, Lang U and Klaritsch P: Placenta praevia: Incidence, risk factors and outcome. J Matern Fetal Neonatal Med 29: 1395-1398, 2016.

22. Cresswell JA, Ronsmans C, Calvert C and Filippi V: Prevalence of placenta praevia by world region: A systematic review and meta-analysis. Trop Med Int Health 18: 712-724, 2013.

23. Cheng YW, Eden KB, Marshall N, Pereira L, Caughey AB and Guise JM: Delivery after prior cesarean: Maternal morbidity and mortality. Clin Perinatol 38: 297-309, 2011.

24. Wu Y, Kataria Y, Wang Z, Ming WK and Ellervik C: Factors associated with successful vaginal birth after a cesarean section: A systematic review and meta-analysis. BMC Pregnancy Childbirth 19: 360, 2019.

25. Rowe R, Li Y, Knight M, Brocklehurst P and Hollowell J: Maternal and perinatal outcomes in women planning vaginal birth after caesarean (VBAC) at home in England: Secondary analysis of the birthplace national prospective cohort study. BJOG 123: 1123-1132, 2016.

26. Macdorman MF, Declercq E, Mathews TJ and Stotland N: Trends and characteristics of home vaginal birth after cesarean delivery in the United States and selected States. Obstet Gynecol 119: 737-744, 2012.

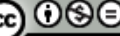

This work is licensed under a Creative Commons Attribution-NonCommercial-NoDerivatives 4.0 International (CC BY-NC-ND 4.0) License. 